\title{
Arqueoastronomía en el Sitio Arqueológico de Yarumela
}

Josué Erubel Ramos Castro ${ }^{1}$

\section{RESUMEN}

El Sitio Arqueológico de Yarumela es considerado como uno de los sitios prehispánicos más antiguos del corredor mesoamericano en honduras, tiene una existencia casi de aproximadamente 3200 años, hoy en día se encuentra localizado entre los departamentos de Comayagua y La Paz. Se cree que los habitantes que ocuparon el sitio en su momento más asombroso tenían un conocimiento avanzado en cuanto a observación de los cuerpos celestes. En esta investigación se consideró específicamente los movimientos aparentes del sol sobre el horizonte oeste para determinar el alineamiento entre las estructuras en los días de los equinoccios y solsticios. Según las investigaciones realizadas por arqueólogos este sitio fue ocupado durante el período formativo (1000 a.C. a 250 d.C) donde se desarrolló una sociedad indígena que formó la base de la cultura Lenca encontrada por los conquistadores en 1537 (Dixon, Boyd, 1991) citado en (Ramos , 2014,en prensa). La metodología utilizada fue medir el acimut de la puesta del sol por el horizonte y el acimut de las estructuras como también la altura de la montaña específicamente por donde se oculta el sol y así determinar la orientación y alineamientos de las estructuras con respecto al sol.

Palabras clave: Arqueoastronomía, cultura, alineamientos, equinoccios y solsticios

1 Profesor del Departamento de Arqueoastronomía y Astronomía Cultural, Facultad de Ciencias Espaciales, UNAH: jeramos@unah.edu.hn 


\section{ABSTRACT}

The Archaeological Site of Yarumela is considered one of the oldest pre-Hispanic sites of the Mesoamerican corridor in Honduras, has an existence of approximately 3200 years, today it is located between the departments of Comayagua and La Paz. It is believed that the inhabitants who occupied the site at its most amazing moment had an advanced knowledge regarding observation of celestial bodies. In this investigation, the apparent movements of the sun over the western horizon were specifically considered to determine the alignment between the structures on the days of the equinoxes and solstices. According to the researches carried out by archaeologists, this site was occupied during the formative period (1000 BC to $250 \mathrm{AD}$ ) where an indigenous society developed that formed the basis of the Lenca culture found by the conquerors in 1537 (Dixon, Boyd, 1991) cited in (Ramos, 2014, in press). The methodology used was to measure the azimuth of the sunset by the horizon and the azimuth of the structures as well as the height of the mountain specifically where the sun is hidden and thus determine the orientation and alignment of the structures with respect to the sun.

Keywords: Archaeoastronomy, culture, alignments, equinoxes and solstices 


\section{INTRODUCCIÓN}

Esta investigación surge como iniciativa a partir de los hallazgos encontrados en el Sitio Arqueológico de Yarumela y de los resultados de la investigación de la primera fase del mismo, es claro que se puede ir más a fondo en este lugar tan sorprendente y que realmente muestra indicios de que todas sus estructuras tenían un significado desde el punto de vista Arqueoastronómico producto de que la cultura que vivió en el lugar ya reconocida por muchos arqueólogos que analizaron el sitio arqueológico mencionan sus trascendencia cultural en Mesoamérica desde el periodo formativo (1000 a.C) y periodo tardío (400 a.C). Hay una enorme posibilidad de encontrar sitios arqueológicos en donde se puede interpretar registros, desde una perspectiva Arqueoastronómica.

En la investigación de la primera fase y otras de tipo reconocimiento, se logró georreferenciar el sitio con algunas estructuras, medir la altura de las mismas, el acimut entre las estructuras, el acimut de la salida y las puestas del sol para los equinoccios y solsticios, también se analiso la cobertura de suelo cercana al sitio arqueológico con el propósito de conocer su estado actual en cuanto a conservación del mismo. El sitio de Yarumela está prácticamente abandonado desde el punto de vista de protección del patrimonio, Hoy en día en este lugar se desarrollan actividades de la vida cotidiana, poniendo en peligro las evidencias de esta cultura. Existe interés de parte del Departamento de Arqueoastronomía y Astronomía Cultural por apoyar a través de las investigaciones, la protección del patrimonio cultural de nuestro país y hacer actividades de vinculación en la comunidad de Miravalle y Yarumela, vecinos cercanos al Sitio Arqueológico para seguir despertando el interés de protegerlo con el propósito de dar un sentido cultural, económico y turístico.

\section{METODOLOGÍA}

El enfoque de esta investigación es cuantitativo porque busca resolver un problema basado en el análisis de la orientación de las estructuras, consideradas importantes para el estudio. El trabajo de investigación consistió en medir dos variables acimut de la estructura, acimut de la puesta del sol por el horizonte y altura de las montañas en el horizonte punto cardinal oeste, por donde el sol se pone en los diferentes eventos solares equinoccios y solsticios. Estas mediciones se realizaron con aparatos y herramientas especiales como estación total para medir altura del horizonte y acimut entre 
estructuras. Todos estos datos fueron introducidos en un programa diseñado por el autor utilizando un algoritmo simple de cálculo de trigonometría donde se pudo determinar si estas estructuras tienen una relación Arqueoastronomica. Se realizo una comparación de los resultados obtenidos con el software de simulación virtual stellarium y de esta manera ratificar que los cálculos realizados estarían en un rango aceptable de alineamientos entre las estructuras para los años 400 a.C y 2015 d.C.

\section{RESULTADOS}

\section{Sitio Arqueológico Yarumela}

Durante el período formativo (1000 a.C. a 250 d.C) en el centro de Honduras en el sitio arqueológico de Yarumela, se desarrolló una sociedad indígena que formó la base de la cultura Lenca encontrada por los conquistadores en 1537. (Dixon B. , 1991). Los hallazgos encontrados en el Sitio Arqueológico de Yarumela el cual corresponde al periodo clásico formativo mesoamericano y que fue pronto reconocido por ciertos investigadores porque en este lugar hubo un contacto temprano entre Mesoamérica y Perú, donde florecieron las culturas como la olmeca y la chavín respetivamente hace más de 1,500 años a.C. Este sitio es conocido localmente como Chilcal y según las investigaciones tenían un conocimiento avanzado que podemos confirmar en esta investigación en cuanto a la observación del cielo a simple vista. Se cree que Yarumela era el asentamiento del cacicazgo dominante de todo el valle durante los tiempos del formativo tardío 400 a.C. Hasta por lo menos 250 años d.C. (Dixon 1989) citado en (Mandeville J. L., 1997)Finalmente la arquitectura monumental en tal escala nunca fue practicada después del siguiente período clásico y eventualmente fue abandonada por completo afínales del período postclásico, Dixon 1989 citado en (Mandeville J. L., 1997).

Ubicación geográfica de Yarumela El emplazamiento arqueológico Yarumela está ubicado en el valle de Comayagua que se extiende por $1.5 \mathrm{~km}$ y una altitud de 600 metros sobre el nivel del mar. Está asentado en la ribera occidental del Río Humuya. Las coordenadas geográficas del Sitio Arqueológico de Yarumela Son: Latitud $14.3637^{\circ} \mathrm{N}$ y Longitud $87.65^{\circ} \mathrm{W}$.

Para comprender mejor la ubicación del sitio se elaboró mapas georreferenciados y de un posible acercamiento a la cobertura de suelo del año 2003 del Sitio Arqueológico de Yarumela con los puntos geográficos de las estructuras más importantes para 
este estudio, estos puntos fueron tomados con GPS, en el mismo se puede observar que las estructuras 101 y 102 están orientadas de este a oeste y las 103,102 y 104 de sur a norte. Hay otros montículos o estructuras que fueron tomadas en consideración como la 106 y 108 111, 110, 107, 109 y 105 (Ver Fig. 1, Fig.2 y tabla 1).

Figura. 1 Mapa de distribución espacial de las estructuras del grupo principal del sitio arqueológico de yarumela. Fuente elaboración propia.

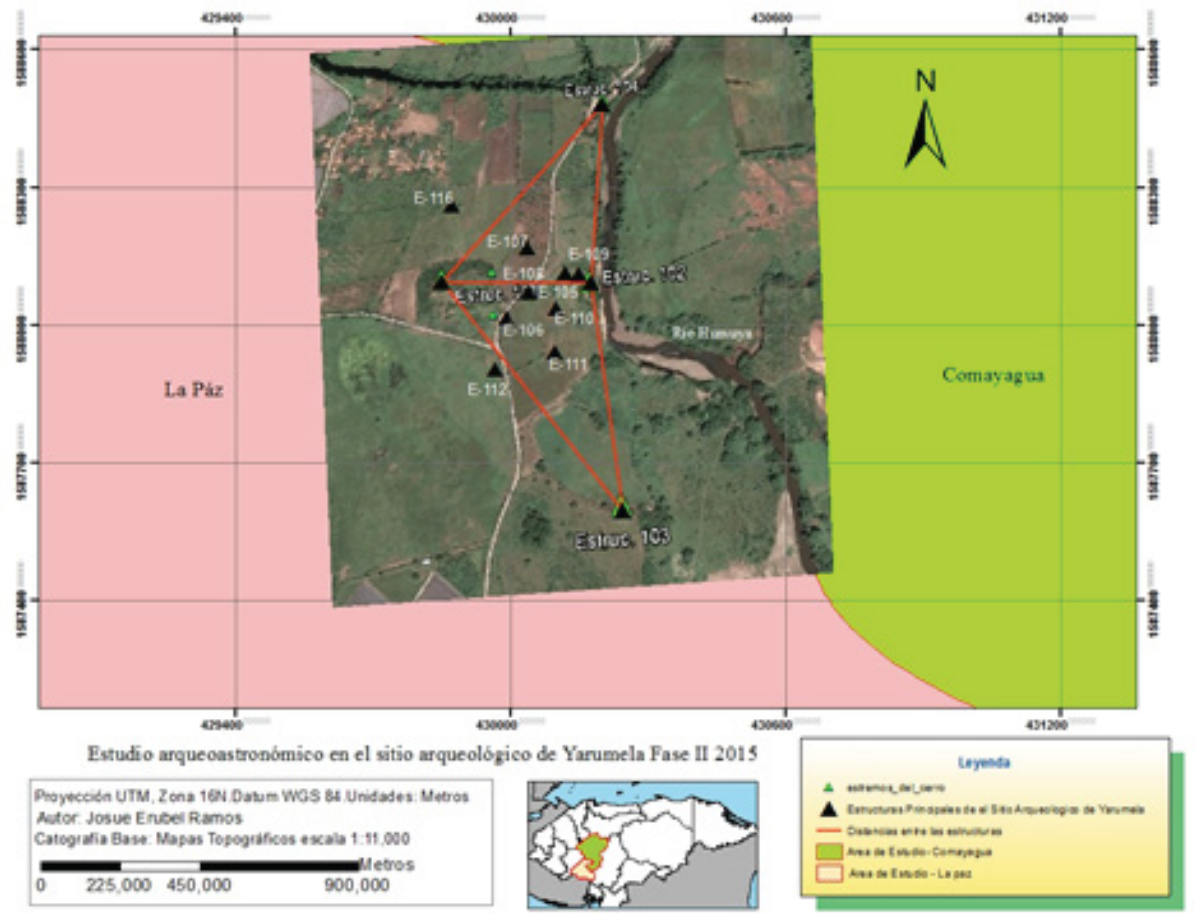

Se tomó fotos en el momento en el que el sol se pone por el horizonte para determinar la Arqueoastronomía y el significado de la posición horizontal de la puesta del sol en relación con un particular alineamiento de los montículos durante los solsticios y equinoccios Ver Fig. 3, 4, 5 y 6. 
Figura. 2. Mapa del Sitio Arqueológico de Yarumela, con cobertura de suelo para el año 2003.Fuente Elaboración propia.

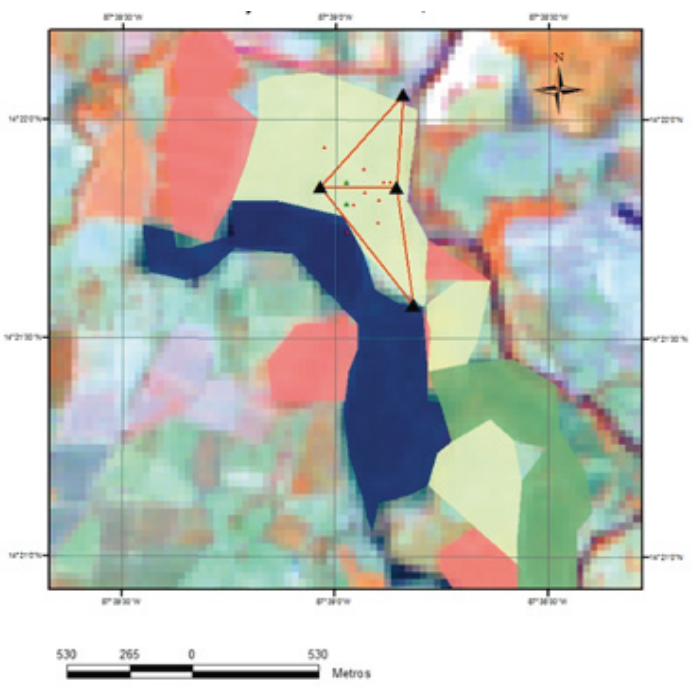

\section{LEYENDA}

4 Estremo sur y norte de la estructura \# 101

- Estructuras mas pequeñas

A Estructuras Principales de mayor altura

Distancias entre las estructuras

Matorra I

Áreas vegetales menos densas

Pasto

Medidas de humedad de la ve getación

Imagenes Landsat TM+ UTM WGS 1984

Tegucigalpa, Honduras

Autor: Josue Erubel Ramos

Septiembre 2015

Tabla 1. Coordenadas geográficas de cada una de las estructuras.

\begin{tabular}{|c|c|c|c|}
\hline Estructuras & Latitud & Longitud & $\begin{array}{l}\text { Altura de la } \\
\text { estructura }\end{array}$ \\
\hline 101 & $14^{\circ} 21^{\prime} 49.20304^{\prime \prime} \mathrm{N}$ & $87^{\circ} 39^{\prime} 2.999^{\prime \prime} \mathrm{W}$ & $20 \mathrm{~m}$. \\
\hline 102 & $14^{\circ} 21^{\prime} 49.3560^{\prime \prime} \mathrm{N}$ & $87^{\circ} 38^{\prime} 51.26568^{\prime \prime} \mathrm{W}$ & $9 m$ \\
\hline 103 & $14^{\circ} 21^{\prime} 35.07976^{\prime \prime} \mathrm{N}$ & $87^{\circ} 38^{\prime} 48.84185^{\prime \prime} \mathrm{W}$ & $6 m$ \\
\hline 104 & $14^{\circ} 22^{\prime} 3.95220^{\prime \prime} \mathrm{N}$ & $87^{\circ} 38^{\prime} 50.456642^{\prime \prime} \mathrm{W}$ & $6 m$ \\
\hline 106 & $14^{\circ} 21^{\prime} 48.2822^{\prime \prime} \mathrm{N}$ & $87^{\circ} 38^{\prime} 57.44038^{\prime \prime} \mathrm{W}$ & $4 \mathrm{~m}$. \\
\hline 108 & $14^{\circ} 21^{\prime} 50.05940 " \mathrm{~N}$ & $87^{\circ} 38^{\prime} 55.8635^{\prime \prime} \mathrm{W}$ & $3 m$ \\
\hline 111 & $14^{\circ} 21^{\prime} 45.88626^{\prime \prime} \mathrm{N}$ & $87^{\circ} 38^{\prime} 53.9417^{\prime \prime} \mathrm{W}$ & $\begin{array}{c}\text { No se conoce } \\
\text { su altura }\end{array}$ \\
\hline & $14^{\circ} 21^{\prime} 54.1644^{\prime \prime} \mathrm{N}$ & $87^{\circ} 38^{\prime} 56.67345 \mathrm{~W}$ & $\begin{array}{c}\text { No se conoce } \\
\text { su altura }\end{array}$ \\
\hline
\end{tabular}

Fuente: Elaboración propia. 
Figura. 3. Estructura 101 vista desde la estructura 102

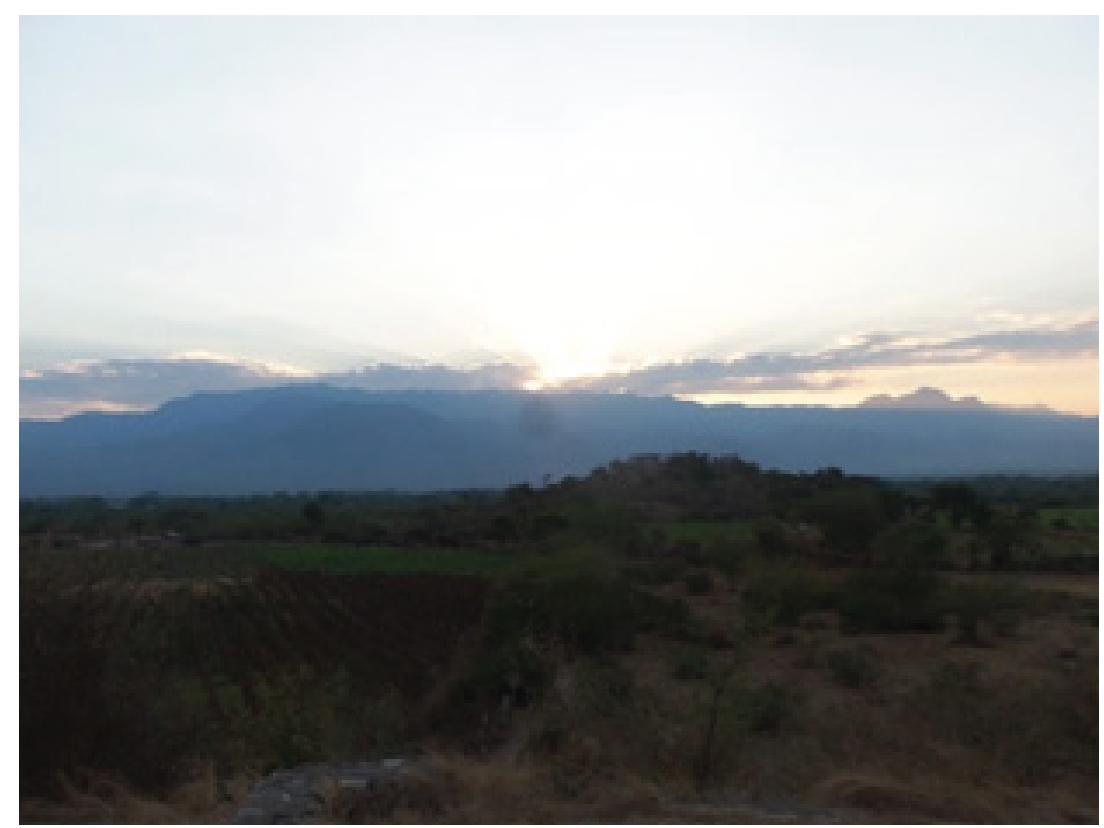

Figura. 4. Puesta del sol en el equinoccio de primavera 20 de marzo 2015

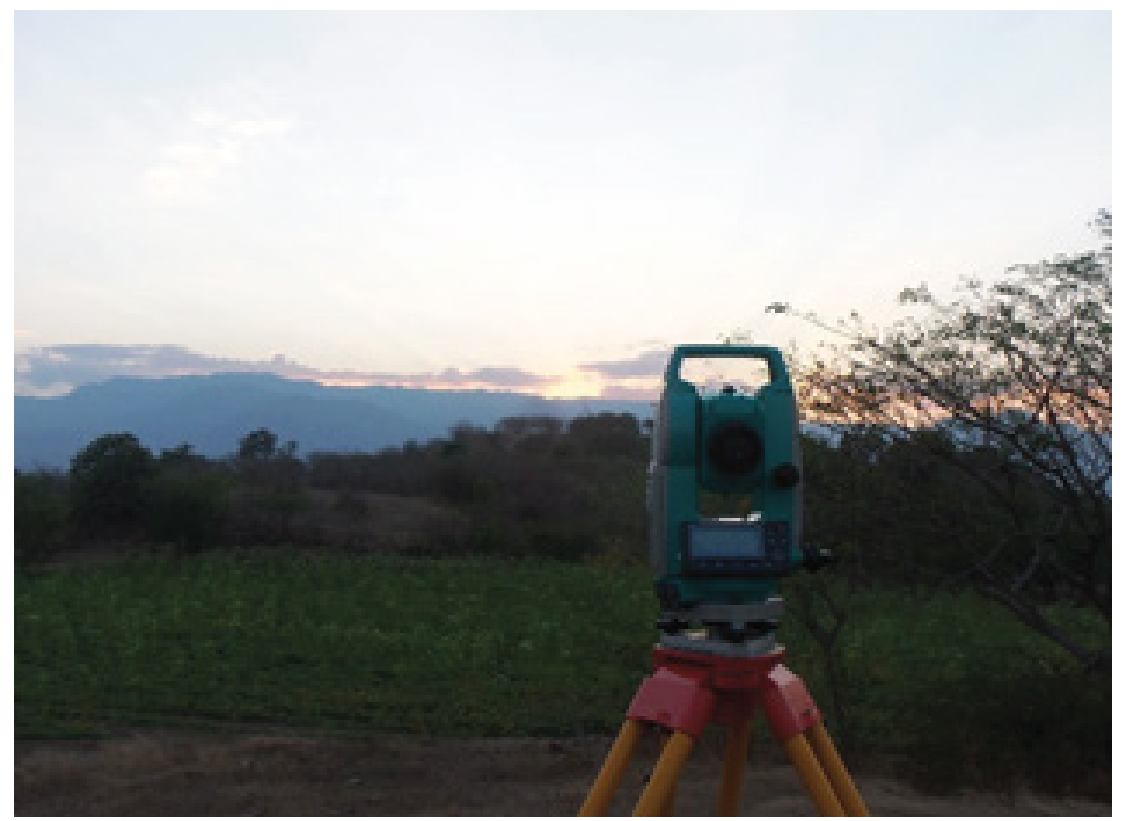


Figura. 5. Puesta del sol en el Solsticio de invierno 21 de diciembre 2015. Vista desde la estructura 108

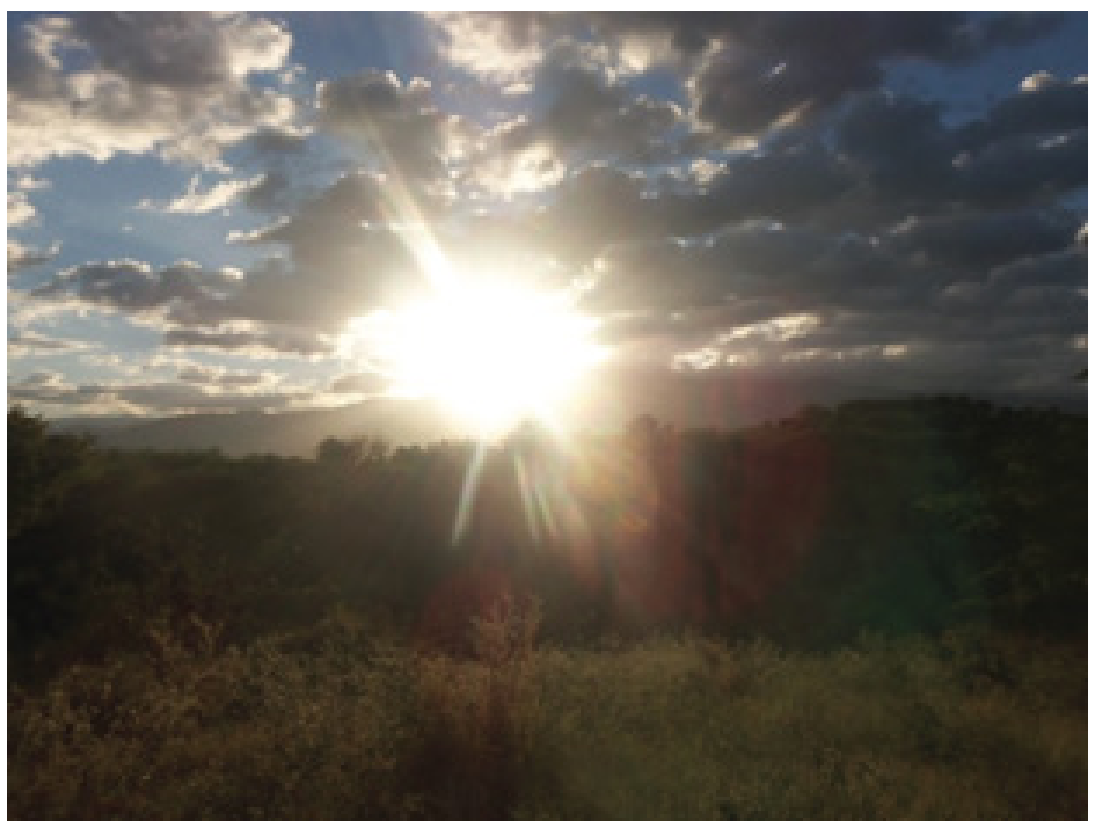

Figura. 6. Puesta del Sol en el solsticio de verano 21 de junio del 2015. Vista desde la estructura 108

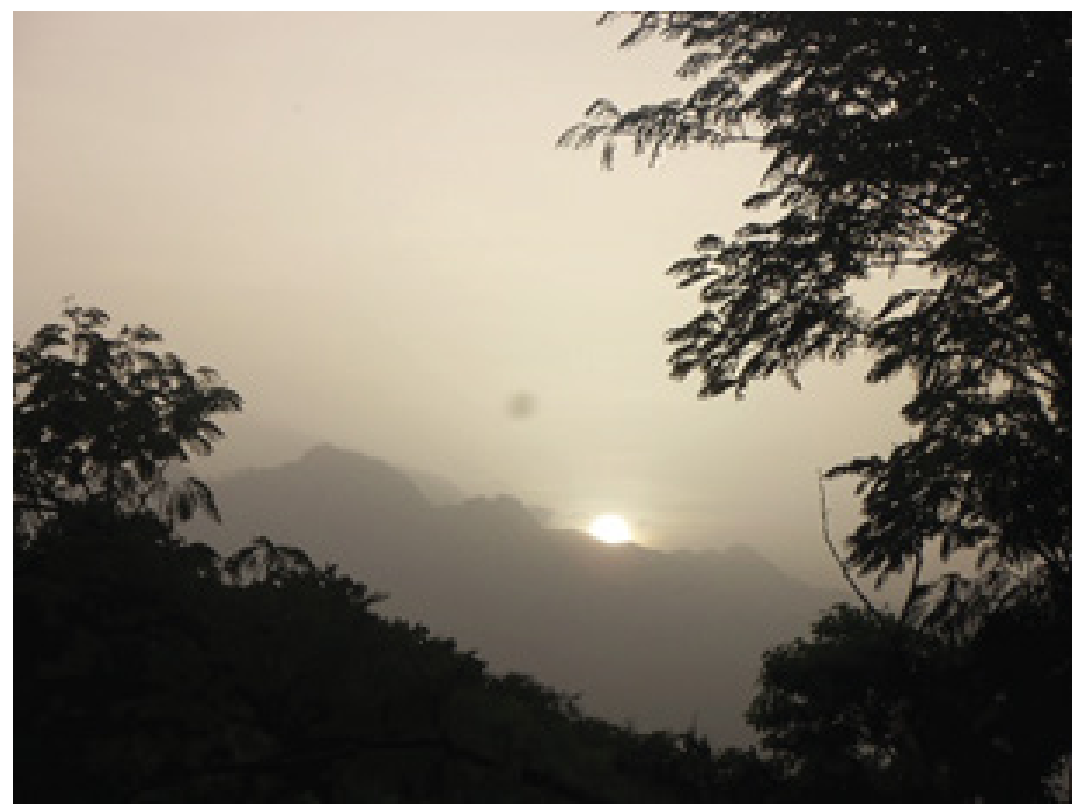


Tabla 2. Acimut entre estructuras y la distancia entre ellas y Angulo de elevación del sol sobre las montañas. Fuente Elaboración Propia

\begin{tabular}{|c|c|c|c|c|}
\hline Estructura & Acimut & Distancia en metros & $\begin{array}{l}\text { Ángulo de } \\
\text { Elevación }\end{array}$ & Análisis \\
\hline $102-104$ & $01^{\circ} 59^{\prime} 57^{\prime \prime}$ & $457.07 \mathrm{~m}$ & & \\
\hline $102-103$ & $169^{\circ} 27$ '45 " & $439.50 \mathrm{~m}$ & & \\
\hline $102-111$ & $219^{\circ} 36^{\prime} 13^{\prime \prime}$ & $123.31 \mathrm{~m}$ & & \\
\hline $102-112$ & $237^{\circ} 26^{\prime} 33^{\prime \prime}$ & $248 m$ & & \\
\hline $102-110$ & $245^{\circ} 35^{\prime} 39^{\prime \prime}$ & $90.19 \mathrm{~m}$ & & \\
\hline $102-106$ & $264^{\circ} 00^{\prime} 05^{\prime \prime}$ & $188.98 \mathrm{~m}$ & & \\
\hline $102-101$ & $272^{\circ} 56^{\prime} 33^{\prime \prime}$ & $323.65 \mathrm{~m}$ & & Alineamiento \\
\hline $102-108$ & $281^{\circ} 37^{\prime} 37^{\prime \prime}$ & $140.98 \mathrm{~m}$ & & \\
\hline $102-107$ & $311^{\circ} 31^{\prime} 33^{\prime \prime}$ & $218.00 \mathrm{~m}$ & & \\
\hline $102-109$ & $316^{\circ} 57^{\prime} 58^{\prime \prime}$ & $58.73 \mathrm{~m}$ & & \\
\hline $102-105$ & $346^{\circ} 44^{\prime} 24^{\prime \prime}$ & $38.61 \mathrm{~m}$ & & \\
\hline 108-SOL & $295^{\circ} 04^{\prime} 10$ & ------------0------------- & $4^{\circ} 15^{\prime} 14^{\prime \prime}$ & Alineamiento \\
\hline 108-SOL & $266^{\circ} 52^{\prime} 05^{\prime \prime}$ & ------------0------------ & $6^{\circ} 24^{\prime} 14^{\prime \prime}$ & Alineamiento \\
\hline 108-SOL & $242^{\circ} 38^{\prime} 16^{\prime \prime}$ & ------------0---------- & $5^{\circ} 56^{\prime} 28^{\prime \prime}$ & Alineamiento \\
\hline
\end{tabular}

Fuente: Elaboración propia.

Se utilizó una estación total para medir la altura de la montaña por donde sale el sol en el día del equinoccio de primavera, otoño y solsticio de verano e invierno. Ver tabla 3 y Fig. 7 
Tabla 3. Altura de la montaña en Grados Minutos y segundos, por donde se pone el sol

\begin{tabular}{|c|c|c|c|c|}
\hline Lugar & \multicolumn{4}{|c|}{$\begin{array}{c}\text { Sitio Arqueológico de Yarumela } \\
\text { (alturas de la montaña en el oeste) }\end{array}$} \\
\hline $\begin{array}{c}\text { Evento } \\
\text { solar }\end{array}$ & $\begin{array}{c}\text { Equinoccio } \\
\text { de Primavera }\end{array}$ & $\begin{array}{c}\text { Solsticio } \\
\text { de verano }\end{array}$ & $\begin{array}{c}\text { Equinoccio } \\
\text { de otoño }\end{array}$ & $\begin{array}{c}\text { Solsticio de } \\
\text { invierno }\end{array}$ \\
\hline Altura & $6^{\circ} 24^{\prime} 14^{\prime \prime}$ & $4^{\circ} 15^{\prime} 14^{\prime \prime}$ & $6^{\circ} 24^{\prime} 14^{\prime \prime}$ & $5^{\circ} 56^{\prime} 38^{\prime \prime}$ \\
\hline
\end{tabular}

Fuente: Elaboración propia.

Figura 7. Plano topográfico del Sitio Arqueológico de Yarumela.

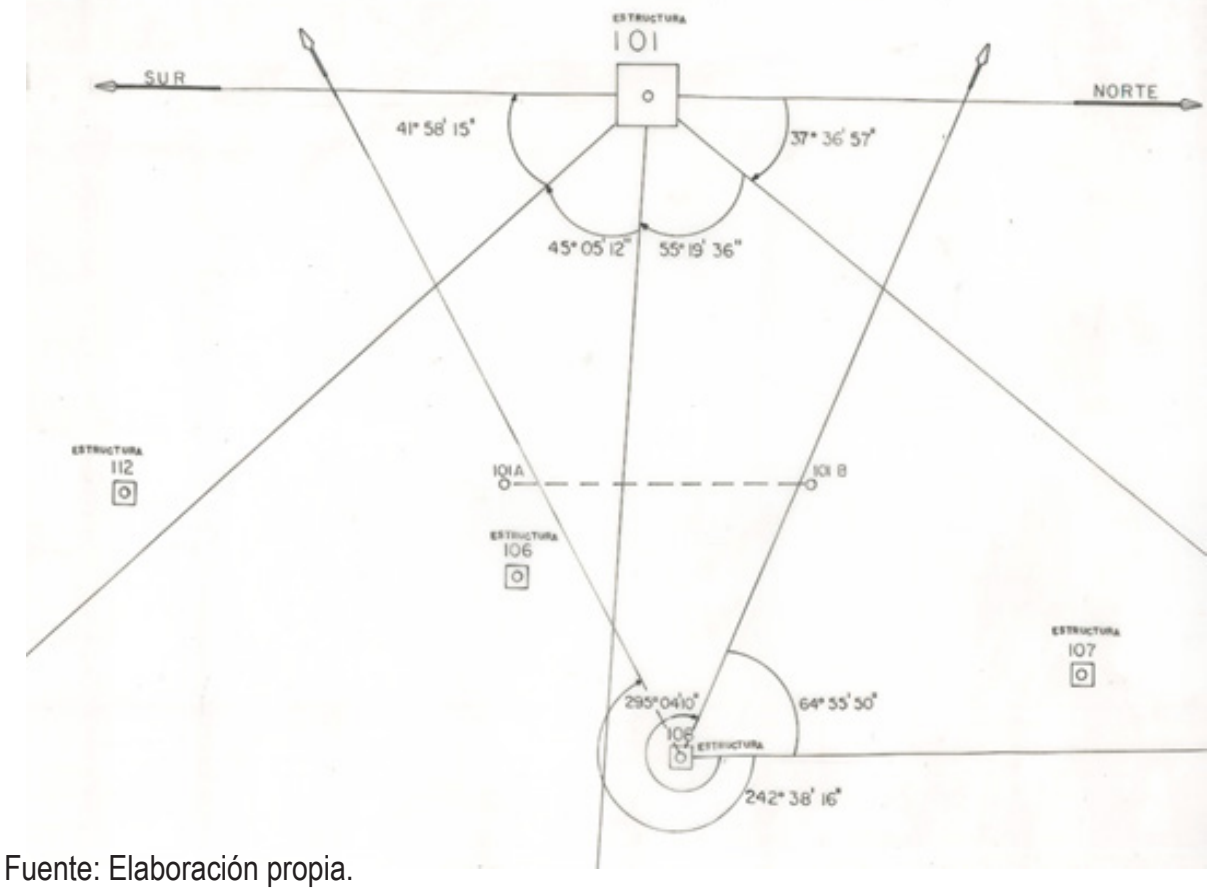

El Stellarium es un software que permite a las personas simular un planetario en su propia computadora, es software libre y está disponible para los principales sistemas. Se comparo los años 400 a.C. y 2015 d.C para observar la similitud con los cálculos., 
considerando que sin montañas el Horizonte es perfecto, los equinoccios están muy cerca de los $270^{\circ}$, el solsticio de invierno de $246.5^{\circ}$ y el solsticio de verano $293.5^{\circ}$ de acimut.

Las siguientes figuras muestran la similitud de la puesta del sol en los días de equinoccio y que están muy cercanos a resultados de acimut medias con estación total Ver. Fig. 8,9 y tabla 4.

Figura 8. Imagen del software stellarium día del equinoccio de primavera año 400 a.C

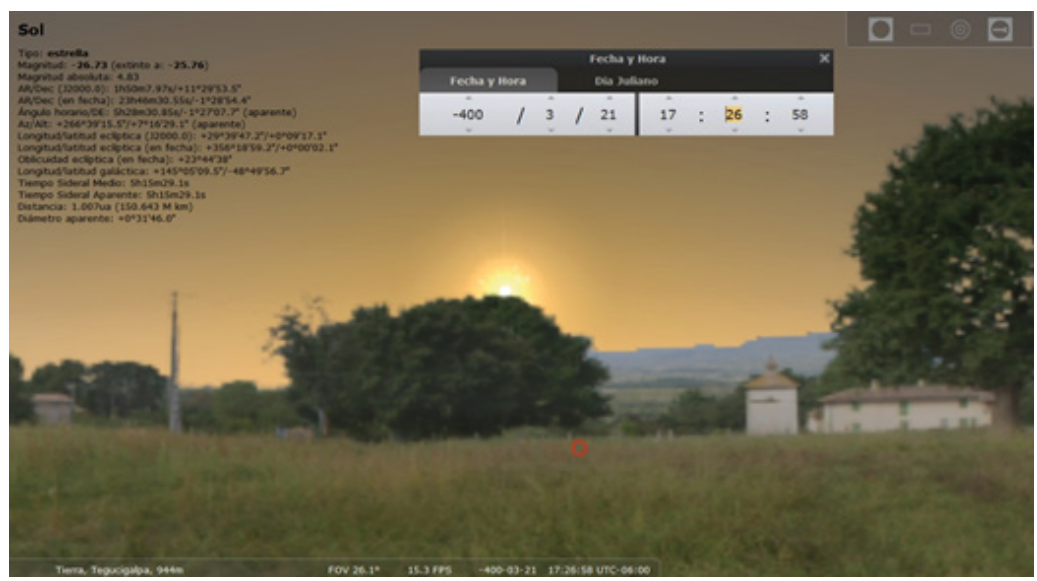

Fuente: Elaboración propia.

Figura 9. Imagen del software stellarium día del equinoccio de primavera año 2015 d.C

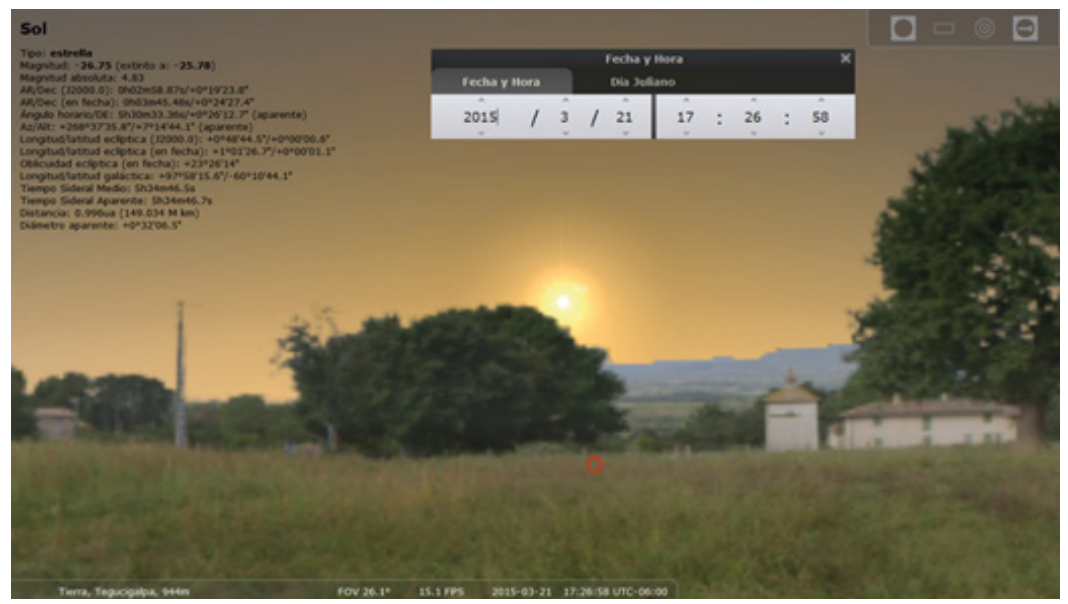

Fuente: Elaboración propia. 


\section{Tabla 4. Comparación de cálculos de acimut, entre el sol y las estructuras con estación total y simulador stellarium}

\begin{tabular}{|c|c|c|c|c|c|}
\hline $\begin{array}{l}\text { Evento } \\
\text { solar }\end{array}$ & $\begin{array}{l}\text { Cálculo de } \\
\text { acimut del } \\
\text { sol con } \\
\text { estación } \\
\text { total en } \\
\text { grados }\end{array}$ & $\begin{array}{l}\text { Acimut de } \\
\text { las } \\
\text { estructuras }\end{array}$ & $\begin{array}{l}\text { Estructuras } \\
\text { relacionadas }\end{array}$ & $\begin{array}{c}\text { Acimut } \\
\text { calculado con } \\
\text { stellarium } \\
\text { años } 2015 \text { Y - } \\
400\end{array}$ & Observaciones \\
\hline $\begin{array}{l}\text { Equinoccio } \\
\text { de } \\
\text { primavera }\end{array}$ & $\begin{array}{l}267.434^{\circ} \\
266.868^{\circ}\end{array}$ & $272.9425^{\circ}$ & $\begin{array}{l}102-101 \\
108-101\end{array}$ & $\begin{array}{l}269.29^{\circ}(2015) \\
267.52^{\circ}(-400)\end{array}$ & $\begin{array}{c}\text { Se observa una } \\
\text { alineación muy } \\
\text { cercana a los } 270^{\circ} \\
\text { en la puesta del sol } \\
\text { observando desde } \\
\text { dos estructuras } 102 \\
\text { y } 108 \text { hacia la } 101\end{array}$ \\
\hline $\begin{array}{l}\text { Solsticio de } \\
\text { verano }\end{array}$ & $\begin{array}{l}295.06^{\circ} \\
295.06^{\circ}\end{array}$ & $295^{\circ} 04^{\prime} 10^{\prime \prime}$ & $\begin{array}{l}108-S O L \\
108-101 B\end{array}$ & $\begin{array}{l}293.16^{\circ}(2015) \\
293.33^{\circ}(-400)\end{array}$ & $\begin{array}{c}\text { Desde la estructura } \\
101 \text { y la puesta del } \\
\text { sol medida desde la } \\
\text { estructura } 108 \text { hay } \\
25.06^{\circ} \text { grados al } \\
\text { norte cercano a la } \\
\text { declinación norte de } \\
+23.5^{\circ} \text { y también } \\
\text { coincide } \\
\text { aproximadamente } \\
\text { con la esquina } \\
\text { inferior derecha de } \\
\text { la estructura } 101- \\
\text { (101B) }\end{array}$ \\
\hline $\begin{array}{l}\text { Equinoccio } \\
\text { de otoño }\end{array}$ & $\begin{array}{l}267.434^{\circ} \\
266.868^{\circ}\end{array}$ & $272.9425^{\circ}$ & $\begin{array}{c}102 \text { y } 101 \\
108-101\end{array}$ & $\begin{array}{l}269.29^{\circ}(2015) \\
267.52^{\circ}(-400)\end{array}$ & $\begin{array}{c}\text { Se observa una } \\
\text { alineación muy } \\
\text { cercana a los } 270^{\circ} \\
\text { en la puesta del sol } \\
\text { observando desde } \\
\text { dos estructuras } 102 \\
\text { y } 108 \text { hacia la } 101\end{array}$ \\
\hline $\begin{array}{l}\text { Solsticio de } \\
\text { invierno }\end{array}$ & $242.6^{\circ}$ & $242^{\circ} 38^{\prime} 16^{\prime \prime}$ & $\begin{array}{l}\text { 108-SOL } \\
108-101 \mathrm{~A}\end{array}$ & $\begin{array}{c}244^{\circ}(2015) \\
243.7^{\circ}(-400)\end{array}$ & $\begin{array}{l}\text { Desde la estructura } \\
101 \text { y la puesta del } \\
\text { sol hay } 27.5^{\circ} \\
\text { grados al sur } \\
\text { cercano a la } \\
\text { declinación sur de } \\
--.23 .5^{\circ}\end{array}$ \\
\hline
\end{tabular}




\section{Trigonometría}

El coseno de un lado es igual al producto de los cosenos de los otros dos, más el producto de sus senos multiplicado por el coseno del ángulo comprendido (Berrecoso, Ramírez , Enríquez Salamanca, \& Pérez Peña, 2003).

Para determinar los resultados de la medición con la estación total en cuanto al acimut de las estructuras y la salida del sol en el día del equinoccio y los solsticios se aplicó y se desarrolló la siguiente fórmula.

Fórmula

$\operatorname{Cos}(90-)=\operatorname{Cos}(90-\varphi) \cos (90-h)+\operatorname{sen}(90-\varphi)+\operatorname{sen}(90-\varphi) \operatorname{sen}(90-h) \cos z$ Transformando esta fórmula a trigonometría básica de los senos obtenemos.

Sen $=\operatorname{sen} \varphi \operatorname{sen} h+\cos \cos \varphi \cos h \cos z$

Dejando Para cos z y a este se le resta $360^{\circ}$

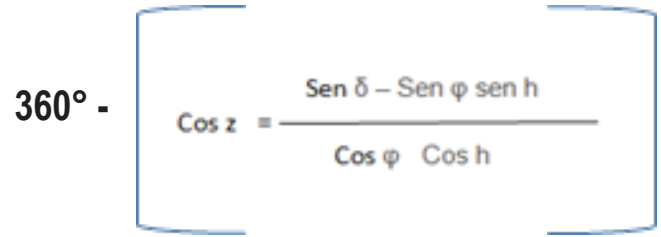

De la formula anterior despegada se desarrolló una aplicación software ver. 1.0.1, para el cálculo del acimut los equinoccios y solsticios. Ver Fig. 10, 11, 12 y 13

\section{Figura 10.}

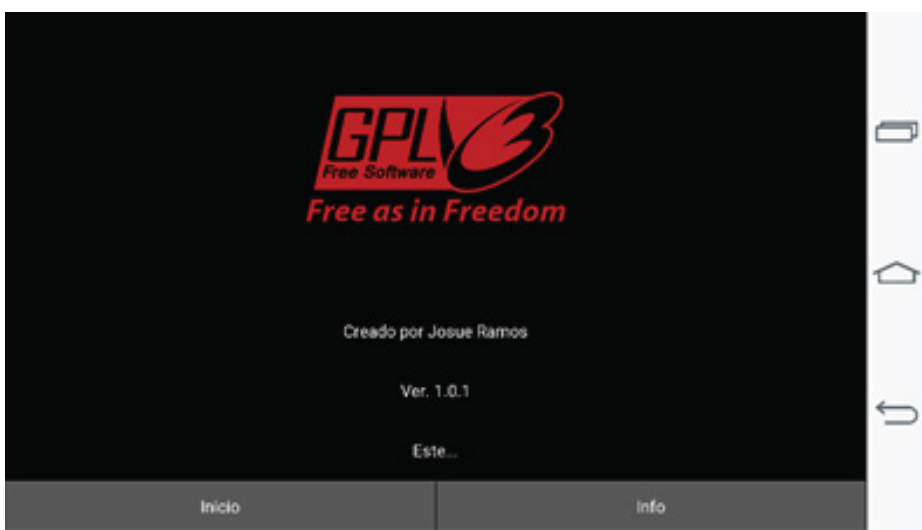

Fuente: Elaboración propia. 


\section{Cálculos para los equinoccios}

Para determinar el acimut de la salida del sol se tomó la altura de la montaña medida desde la estructura 108 que se obtuvo con la estación total y se aplicó y desarrollo la siguiente fórmula para cada uno de los eventos solares.

\section{Datos}

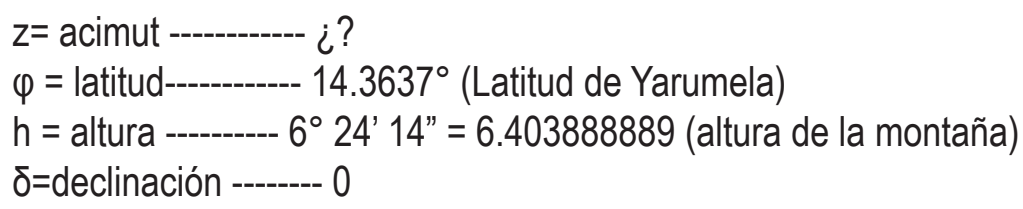

Resultado Z=268.352994894 ${ }^{\circ}$

\section{Figura 11.}

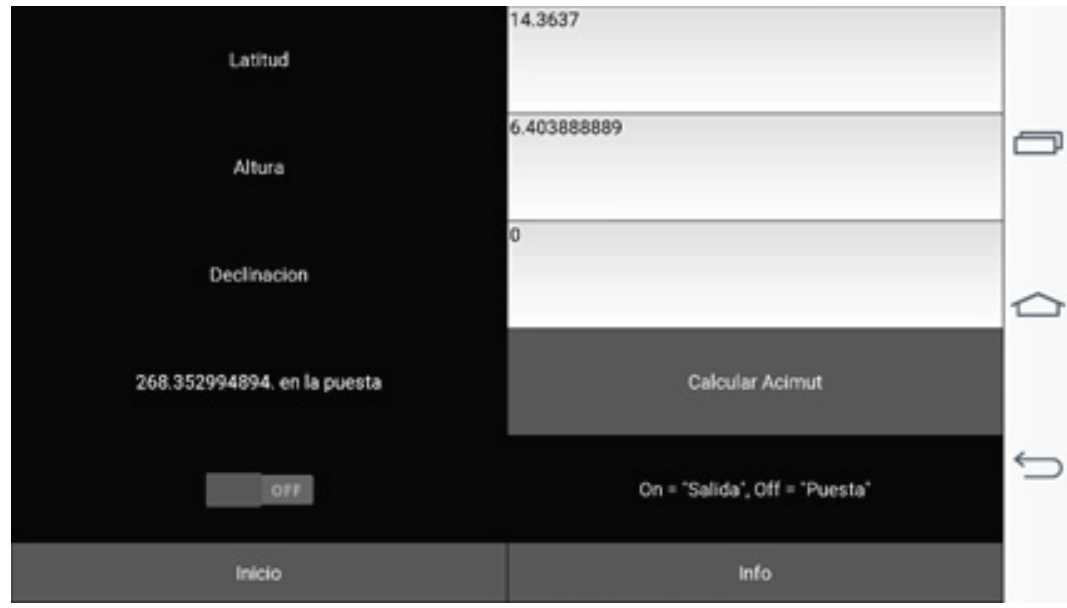

Fuente: Elaboración propia.

\section{Cálculos para el solsticio de verano}

\section{Datos}

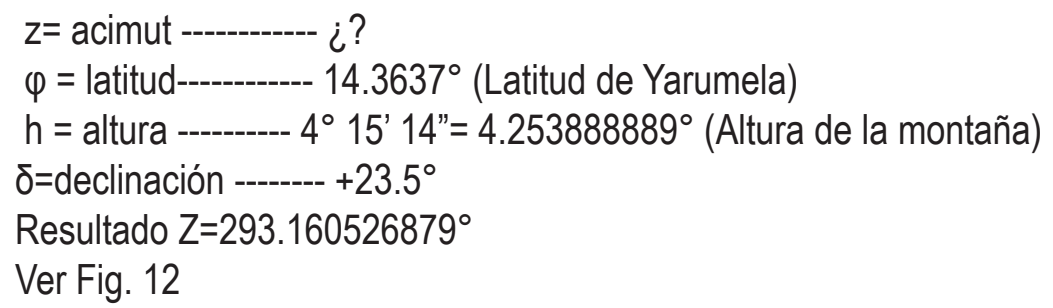




\section{Figura 12.}

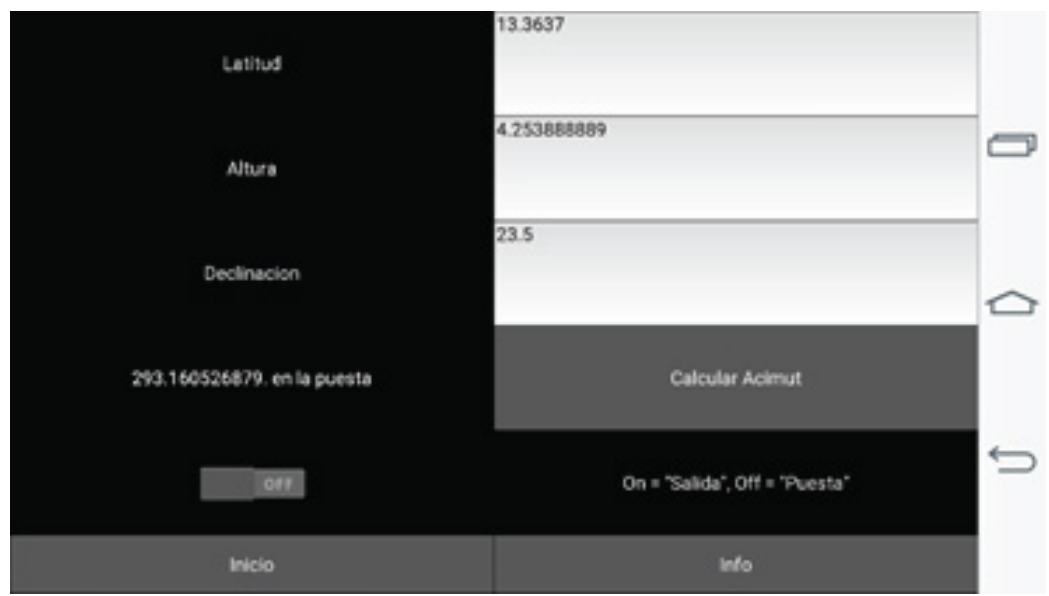

Fuente: Elaboración propia.

Cálculos para el solsticio de invierno

\section{Datos}

$z=$ acimut ---------- $i$ ?

$\varphi=$ latitud----------- $14.3637^{\circ}$ (Latitud de Yarumela)

$h=$ altura ------- $5^{\circ} 56^{\prime} 28^{\prime \prime}=5.9^{\circ}$ (Altura de la montaña)

$\delta=$ declinación ------- $-23.5^{\circ}$

Resultado Z=243.892228596 ${ }^{\circ}$

Ver Fig. 13

\section{Figura 13.}

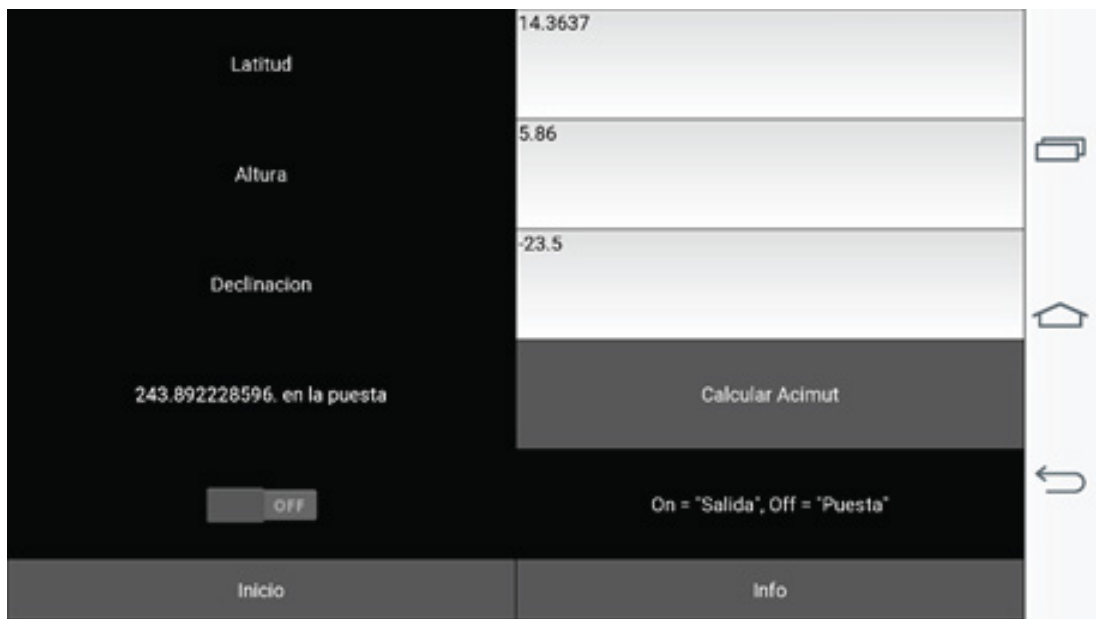

Fuente: Elaboración propia. 


\section{CONCLUSIONES}

1. Para el primer objetivo podemos determinar que entre las estructuras 101 y 102 hay una orientación de este a oeste, y que el sol se pone por encima de las montañas a un acimut muy cercano a los $270^{\circ}$, lo cual nos permite decir que si hay un alineamiento entre estas dos estructuras y la posición del sol en el momento de su puesta por el horizonte en los equinoccios considerando que la variable acimut varía dependiendo de la altura de la montaña.

2. Para el segundo objetivo podemos determinar que las estructuras 101 y las otras estructuras, se está considerando la estructura 108 tanto como punto central de observación para los diferentes eventos solares equinoccios y solsticios. Es importante considerar que los extremos inferiores de la estructura 101(101A y 101B) parecen estar alineadas con los solsticios, en el caso del solsticio de verano parece alinearse la esquina inferior derecha 101B y la estructura 108 y las puestas del sol marcan un acimut muy cercano a los $293.5^{\circ}$ y también la estructura 108 pudo haber sido un punto de observación en los equinoccios ya que desde esta estructura se logra acercar un acimut de $270^{\circ}$ sobre la estructura 101 cuando se pone el sol. Al igual cuando se midió durante el solsticio de invierno desde la estructura 108 a la puesta del sol casi coincidiendo con la esquina inferior izquierda $101 \mathrm{~A}$ de la estructura se forma un ángulo alrededor de los $243^{\circ}$ muy cercano a los $246.5^{\circ}$ donde las alturas de la montaña también inciden en el acimut.

3. Envista de que el objetivo era encontrar alineamientos en el lugar, podemos concluir que si existe una alineación entre las estructuras 101, 102, 108 para los eventos solares y quizás con otras estructuras como la 107, 111 y 110 en las puestas y salidas, pero no se puede determinar a profundidad una orientación o alineación debido a que en el sitio la mayoría de las estructuras están cubiertas por lo cual no se puede determinar sus dimensiones esto fue un obstáculo para la investigación, pero a nuestro parecer la estructura 108 podría haber sido un punto de observación para los eventos solares como equinoccios y solsticios.

4. En cuanto a las comparaciones con el programa del Stellarium del año 400 a. C y 2015 d. C se observa una muy cercana similitud de acimut entre los diferentes eventos solares de equinoccios y solsticios tomando en cuenta que el software no toma la altura de las montañas, solo sirvió como referencia de comparación.

5. La aplicación desarrollada software ver. 1.0.1, se utilizo para introducir los datos y obtener resultados con cálculos mas exactos y que se pueda aplicar en el campo desde un celular, puede ser solicitado al autor al Email jerubel2009@gmail.com 


\section{REFERENCIAS BIBLIOGRÁFICAS}

Berrecoso, M., Ramírez , M. E., Enríquez Salamanca, J. M., \& Pérez Peña, A. (2003). Notas y apuntes de trigonometria esférica y astronomía de posición. (U. d. Cadíz, Ed.)

Dixon, Boyd. (1991). " La Arquitectura del periodo Formativo y la competencia del estado social en Yarumela, Honduras. YAXKIN, XV,edicion extraordinaria 1990-1994, 19-48.

Mandeville, Joesink. (1997). "Programa Arqueologico de Yarumela, temporada 1983: descubrimientos y analisis". YAXKIN, XV, edicion extraordinaria 1990-1994, 5-18.

Ramos , J. E. (2014,en prensa). Estudio Arqueoastronómíco en el Sitio Arqueológico de Yarumela. En prensa.

Ramos Castro, J. E. (2015). Software Acimut Ver. 1.0.1, Software disponible sin propositos comerciales, enviando un e-mail a: Jerubel2009@gmail.com. 